\title{
Association between Prostaglandin-endoperoxide Synthase 2 (PTGS2) Polymorphisms and Blood Pressure in Korean Population
}

\author{
Hyun-Seok Jin', Kyung-Won Hong1, Ji-Eun Lim, \\ Hye-Ree $\mathrm{Han}^{2}$, Jong-Young Lee ${ }^{2}$, Hun Kuk \\ Park ${ }^{1}$ and Bermseok $\mathrm{Oh}^{1 *}$ \\ ${ }^{1}$ Department of Biomedical Engineering, School of \\ Medicine, Kyung Hee University, Seoul 130-701, Korea, \\ ${ }^{2}$ Center for Genome Science, National Institute of \\ Health, Seoul 122-701, Korea
}

\begin{abstract}
Blood pressure refers to the force exerted by circulating blood on the walls of blood vessels, and chronical elevation of blood pressure is known as hypertension. Although hypertension is affected by genetic and environmental factors, the genetic background of hypertension is not fully understood. One of the candidate genetic factors, Prostaglandin-endoperoxide synthase 2 (PTGS2), is a membrane-bound enzyme, catalyzing the conversion of arachidonic acid to prostaglandin, and recently SNPs of PTGS2 gene was associated with hypertension in Japanese population. Therefore the association of PTGS2 polymorphisms was investigated with blood pressure in healthy Korean subjects, 470 unrelated individuals randomly selected from Ansung and Ansan cohorts. The 25 SNPs of PTGS2 gene were identified by the sequencing analysis of 24 Korean samples. Among identified polymorphisms, three SNPs (rs689466, $-1329 \mathrm{~A}>\mathrm{G}$; rs5275, +6365T > C; rs4648308, +8806G > A) were selected for further association analysis, and rs689466 located in promoter region was associated with blood pressure as well as triglyceride level in the blood. By in silico analysis, rs689466 locates in v-Myb transcription factor binding site, and the v-Myb site disappears when the SNP is changed from $A$ to $G$ nucleotide. Individuals with $A / G$ and $G / G$ genotype in rs689466 have higher blood pressure than those with A/A genotype, and the regression p-value is 0.008 for systolic and 0.004 for diastolic blood pressure. In summary, the PTGS2 polymorphism (rs689466) is associated with blood pressure in Asian populations based on this and Japanese studies, shedding light on it as a genetic
\end{abstract}

*Corresponding author: E-mail ohbs@khu.ac.kr Tel +82-2-961-0290, Fax +82-2-961-5515

Accepted 6 September 2008 risk marker of hypertension.

Keywords: PTGS2, blood pressure, hypertension, polymorphism, rs689466

\section{Introduction}

Blood pressure refers to the force exerted by circulating blood on the walls of blood vessels, chronic elevation of blood pressure is known as hypertension. In general hypertension express itself no symptoms, however when long period it lets alone, gives an obstacle to brain, heart and kidney and treatment is necessary in order to escape a fatal complication (Dennis et al., 2004). Although Mendelian traits related to hypertension have large effects in affected individuals (Nakayama et al., 2002; Xu et al., 1999), they only account for a very small fraction of hypertension. The contribution of genetic factors to blood pressure variance is estimated to be about $30 \%$, and the genetic background of essential hypertension is not fully understood (Naber and Siffert, 2004; Park et al., 2008).

Prostaglandin-endoperoxide synthase 2 (PTGS2) is a membrane-bound enzyme that catalyzes the conversion of arachidonic acid to prostaglandin and is associated with biologic events such as injury, inflammation, and proliferation (Hla and Neilson, 1992; Tazawa et al., 1994). PTGS2-mediated prostanoids play an important role in maintaining blood pressure (Anderson et al., 1976; Daniels et al., 1967). Specially the cortical PTGS2derived prostaglandin 12 participates in the pathogenesis of renal vascular hypertension through stimulating renal rennin synthesis and release (Hao and Breyer, 2008). Clinical studies as well as animal studies also demonstrate important roles for PTGS2 in maintaining cardiovascular homeostasis (Zewde and Mattson, 2004; Zhang et al., 2006). PTGS2 is upregulated in animal models of cardiac failure (Abassi et al., 2001; Adderley and Fitzgerald, 1999), and its expression has been detected in heart failure in humans (Wong et al., 1998). PTGS2 gene is located on chromosome 1q25.2-q25.3 (Hla and Neilson, 1992) and its cDNA encodes a 604 amino acid protein.

Recently a large-scale association study in Japanese population revealed the association of PTGS2 poly- 
morphism with hypertension (Iwai et al., 2004). Therefore, the association of PTGS2 polymorphisms was addressed in this study with blood pressure in healthy Korean population.

\section{Methods}

\section{Sequencing analysis for PTGS2 SNP identi- fication}

The genetic variants of PTGS2 gene were identified by the sequencing analyses from 24 unrelated Koreans. PTGS2 genomic region was sequenced comprising all exons, 5'UTR, 3'UTR, up to $1.5 \mathrm{~kb}$ exon-intron boundary regions, and promoter region.

The information for the genomic DNA sequence of PTGS2 was obtained from GenBank database (http:// www.ncbi.nlm,nih.gov/). Genomic regions targeted for sequencing were amplified by polymerase chain reaction (PCR) from genomic DNA of 24 immortalized cell lines generated from unrelated Koreans. Primers for PCR were designed using Primer3 program (http://www-genome. wi.mit.edu/cgi-bin/primer/primer3_www.cgi) (Rozen and Skaletsky, 2000) (Supplemental Table 1). PCR amplified fragments were sequenced on both strands using an $A B I$ Prism 3730 sequencer (Applied Biosystems, Foster City, CA, USA) according to the manufacturer's protocol. The PolyPhred program (http:// droog.gs.washington.edu/polyphred/) was employed to assemble the sequences and identify SNPs (Nickerson et al., 1997).

\section{Subjects}

Two community cohorts (Ansung and Ansan) in South
Korea were initiated from 2001 as major projects for the Korean Health and Genome Study (KHGS) in Korea National Institute of Health $(\mathrm{KNIH})$. The Ansan cohort mostly represents urban community, while the Ansung cohort a rural community. All participants in either cohort are about 40 69 years old, reside within the survey area for at least 6 months, and are mentally and physically healthy. Cohort examinations were accomplished biennially for the prospective studies. Up to date, 2,239 men and 2,779 women in Ansung, and 2,523 men and 2,497 women in Ansan participated in the cohorts.

In this study, 470 unrelated individuals were randomly selected from two community cohorts who were healthy based on medical examination results. The study was approved by the institutional review board of $\mathrm{KNIH}$. All subjects gave written informed consents.

\section{Genotyping}

Among identified polymorphisms from the sequencing analysis of PTGS2 gene, three SNPs (rs689466, -1329A $>$ G; rs5275, +6365T > C; rs4648308, +8806G $>$ A) were selected based on minor allele frequency $(>0.05)$, linkage disequilibrium (LD) status and haplotype tagging status (Fig. 1, Supplemental Table 2).

SNPs were genotyped using amplifying primers and probes designed for TaqMan (Livak, 1999). Primer Express (Applied Biosystems) was used to design both the PCR primers and the MGB TaqMan probes. One allelic probe was labeled with the FAM dye, and the other was labeled with the fluorescent VIC dye. PCRs were run in the TaqMan Universal Master mix without UNG (Applied Biosystems), and with PCR primer concentrations of $900 \mathrm{nM}$ and TaqMan MGB probe at a con-
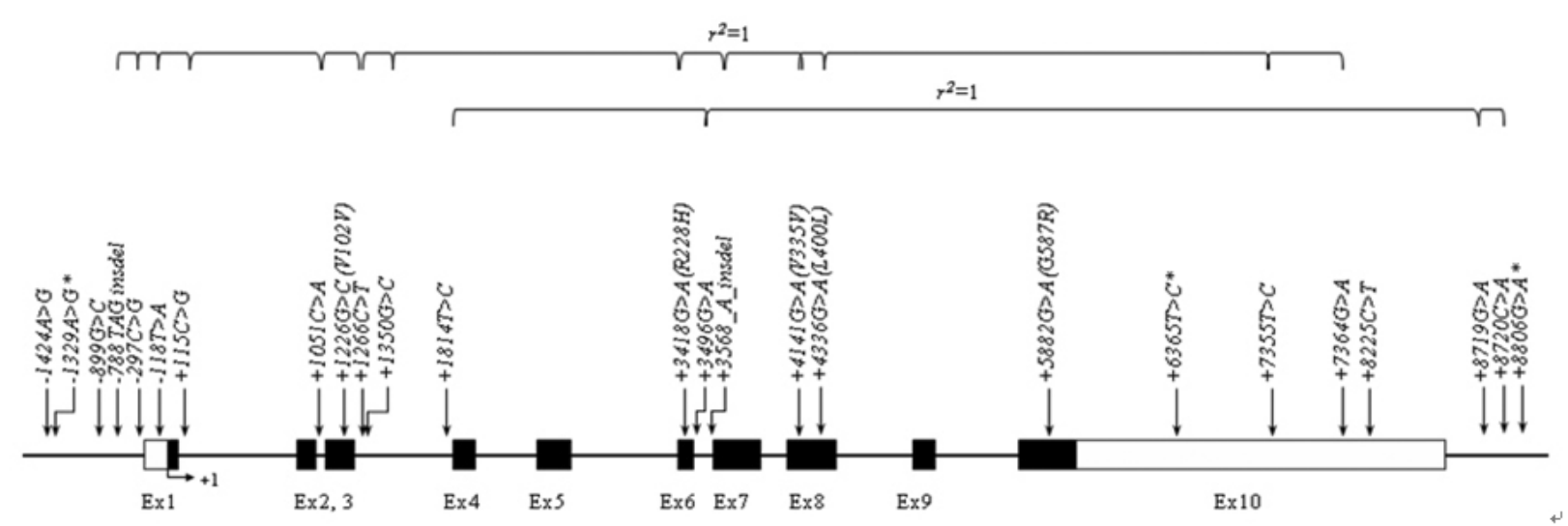

Fig. 1. Map of PTGS2 on chromosome 1q25.2-q25.3 (8.6 kb). Coding exons are marked by shaded blocks and $5^{\prime}$ and $3^{\prime}$ untranslated regions are marked by white blocks. Asterisks $\left(^{*}\right)$ indicate SNPs that were genotyped in the larger population. The first nucleotide of the translational start site is denoted as nucleotide plus one (reference sequence of PTGS2. NC_000001.9). 
Table 1. Association between three SNPs of PTGS2 gene \& dyslipidemia adjusted by age, sex and BMI

\begin{tabular}{|c|c|c|c|c|c|c|}
\hline & \multicolumn{3}{|c|}{ Individual number of genotype (mean $\pm \mathrm{SD} \mathrm{mg} / \mathrm{dL}$ ) } & \multirow{2}{*}{$\frac{\text { Co-dominant }}{p}$} & \multirow{2}{*}{$\frac{\text { Dominant }}{p}$} & \multirow{2}{*}{$\frac{\text { Recessive }}{p}$} \\
\hline & $\mathrm{C} / \mathrm{C}$ & $\mathrm{C} / \mathrm{R}$ & $\mathrm{R} / \mathrm{R}$ & & & \\
\hline \multicolumn{7}{|c|}{ rs689466 (-1329A > G, Promoter) } \\
\hline TG & $132(136.55 \pm 59.05)$ & $233(152.39 \pm 76.58)$ & $102(157.73 \pm 71.36)$ & 0.017 & 0.013 & 0.176 \\
\hline TCHOL & $132(177.97 \pm 29.78)$ & $233(182.1 \pm 31.63)$ & $102(179.85 \pm 34.82)$ & 0.555 & 0.237 & 0.778 \\
\hline HDL & $132(44.05 \pm 10.06)$ & $233(44.43 \pm 9.68)$ & $102(44.55 \pm 10.46)$ & 0.675 & 0.691 & 0.779 \\
\hline LDL & $132(106.61 \pm 27.26)$ & $229(107.83 \pm 27.89)$ & $102(103.76 \pm 28.23)$ & 0.486 & 0.945 & 0.206 \\
\hline \multicolumn{7}{|c|}{ rs5275 $\left(+6365 T>C\right.$, Exon10, $3^{\prime}$ UTR $)$} \\
\hline $\mathrm{TG}$ & $283(151.68 \pm 78.04)$ & $156(146.92 \pm 58.83)$ & $28(133.43 \pm 60.37)$ & 0.126 & 0.191 & 0.226 \\
\hline TCHOL & $283(181.81 \pm 32.33)$ & $156(177.69 \pm 31.6)$ & $28(181.29 \pm 27.44)$ & 0.309 & 0.182 & 0.885 \\
\hline $\mathrm{HDL}$ & $283(44.75 \pm 10.17)$ & $156(43.37 \pm 9.3)$ & $28(45.54 \pm 10.99)$ & 0.616 & 0.345 & 0.511 \\
\hline LDL & $279(107.25 \pm 28.17)$ & $156(104.94 \pm 27.36)$ & $28(109.06 \pm 26.48)$ & 0.706 & 0.478 & 0.623 \\
\hline \multicolumn{7}{|c|}{ rs4648308 (+8806G $>$ A, 3'Downstream) } \\
\hline $\mathrm{TG}$ & $418(149.97 \pm 72.13)$ & $50(143.64 \pm 62.06)$ & $1(81 \pm)$ & 0.371 & 0.437 & 0.329 \\
\hline TCHOL & $418(180.88 \pm 31.54)$ & $50(176.94 \pm 34.03)$ & $1(182 \pm)$ & 0.409 & 0.404 & 0.917 \\
\hline $\mathrm{HDL}$ & $418(44.3 \pm 9.62)$ & $50(44.36 \pm 12.18)$ & $1(60 \pm)$ & 0.615 & 0.778 & 0.112 \\
\hline LDL & $414(106.93 \pm 27.7)$ & $50(103.85 \pm 28.29)$ & $1(105.8 \pm)$ & 0.457 & 0.462 & 0.838 \\
\hline
\end{tabular}

Bold indicated significant result by $\mathrm{p}$-value $<0.05$.

TG: triglyceride, TCHOL: total cholesterol, HDL: high-density lipoprotein, LDL: low-density lipoprotein.

centration of $200 \mathrm{nM}$. Reactions were performed in 384-well format in a total reaction volume of $5 \mu \mathrm{l}$ using $20 \mathrm{ng}$ of genomic DNA. The plates were then placed in a thermal cycler (PE 9700, Applied Biosystems) and heated at $50^{\circ} \mathrm{C}$ for $2 \mathrm{~min}$ and $95^{\circ} \mathrm{C}$ for $10 \mathrm{~min}$, followed by 40 cycles of $95^{\circ} \mathrm{C}$ for $15 \mathrm{~s}$ and $60^{\circ} \mathrm{C}$ for $1 \mathrm{~min}$ with a final soak at $25^{\circ} \mathrm{C}$. The TaqMan assay plates were transferred from the thermal cyclers to a real-time PCR system (Prism 7900HT, Applied Biosystems) that read the fluorescence intensity in each well of the plate. Fluorescence data files from each plate were analyzed using automated software (SDS ver. 2.1, Applied Biosystems).

\section{Clinical characteristic measurement}

Blood samples were drawn for biochemical measurement (triglyceride, total cholesterol, high-density lipoprotein, low-density lipoprotein) and DNA extraction. The clinical characteristics of the study population are shown in Table 1. The blood pressure in this study was measured three times in lying position in systolic and diastolic status, and averaged for three measurements.

\section{Statistics}

Deviation of genotype frequency from expected HardyWeinberg equilibrium was examined with the chi-square test. To approximate a normal distribution, systolic blood pressure, diastolic blood pressure, triacylglyceride, total cholesterol, HDL cholesterol, LDL cholesterol, non
HDL cholesterol, and total/HDL cholesterol ratio were log transformed before analysis (Oh et al., 2007). We examined linkage disequilibrium (representied by $r^{2}$ ) for PTGS2 gene using Haploview v3.2 (http://www.broad. mit.edu/mpg/haploview/) (Barrett et al., 2005). The associations between SNPs and hypertension-related phenotypes were determined by linear regression analysis while controlling age, sex, and BMI. The SAS statistical software package (SAS Institute Inc. Cary, NC, USA) was used to perform general statistical analyses. Statistical significance was determined at a two-tailed value of $p<0.05$.

\section{Korean SNP database}

The information of most SNPs described in this study is available in the Korean SNP database (http://www.ngri. re.kr/SNP/) that was constructed at the Center for Genome Sciences (KNIH).

\section{Results and Discussion}

By direct DNA sequencing in 24 Korean individuals, 25 genetic variants of PTGS2 gene were identified and their allele frequencies were depicted in supplemental Table 2 (refer in detail http://www.ngri.re.kr/SNP/). 6 variants were located in promoter and 5'UTR region, 5 in exons, and 14 in introns and 3'UTR (Fig. 1).

To investigate the association of PTGS2 polymorphisms with blood pressure, 3 SNPs (rs689466, $-1329 \mathrm{~A}>\mathrm{G}$; rs5275, +6365T>C; rs4648308, +8806G > 
A) were selected based on their allele frequencies and tagging of the linkage disequilibrium and were highlighted by bold and asterisk $\left({ }^{*}\right)$ in Fig. 1. Total 470 healthy subjects were selected for the association genotyping from Ansung and Ansan community cohorts in Korea and their clinical profiles were presented in Table 2.

Linear regression analysis was used to examine their associations with blood pressure controlling age, sex and BMI (Table 3). One SNP, rs689466 (-1329A > G) located in promoter was found to be associated with both systolic blood pressure (SBP) and diastolic blood pressure $(\mathrm{DBP})$ in the co-dominant and dominant genetic models (SBP p-value 0.038 and 0.008 in the co-dominant and dominant, respectively, DBP p-value 0.018 and 0.006 in the co-dominant and dominant, respectively). Carriers of $G$ allele in rs689466 showed higher blood pressure in both SBP and DBP than non-carrier (AA genotype). This association result replicates the previous finding in Japan population (Iwai et al., 2004).

Since blood pressure is influenced by the blood, ge-

Table 2. Clinical characteristics of study subjects

\begin{tabular}{lcc}
\hline & $\mathrm{N}$ & MEAN (SD) \\
\hline Age $(\mathrm{yrs})$ & 470 & $63.99(2.87)$ \\
Sex $(\mathrm{M} / \mathrm{F})^{\star}$ & $208 / 262$ & $44.26 / 55.74$ \\
BMI $\left(\mathrm{Kg} / \mathrm{m}^{2}\right)$ & 470 & $23.30(3.11)$ \\
Systolic BP $(\mathrm{mmHg})$ & 470 & $121.01(17.27)$ \\
Diastolic BP $(\mathrm{mmHg})$ & 470 & $75.36(9.86)$ \\
Triacylglyceride $(\mathrm{mg} / \mathrm{dL})$ & 470 & $149.18(71.00)$ \\
Total cholesterol $(\mathrm{mg} / \mathrm{dL})$ & 470 & $180.48(31.73)$ \\
HDL cholesterol $(\mathrm{mg} / \mathrm{dL})$ & 470 & $44.34(9.91)$ \\
LDL cholesterol $(\mathrm{mg} / \mathrm{dL})$ & 466 & $106.61(27.69)$ \\
Non HDL cholesterol $(\mathrm{mg} / \mathrm{dL})$ & 470 & $136.14(30.67)$ \\
Total/HDL cholesterol ratio & 470 & $4.21(0.99)$ \\
\hline
\end{tabular}

*Numbers and frequencies divided by sex. netic effects of PTGS2 polymorphisms on blood traits related to lipid were also investigated. Although no association was detected in total cholesterol, HDL, and LDL analyses, triglyceride levels in these subjects revealed a significant association with rs689466 (Table 1). Carriers of $G$ allele in rs689466 showed higher triglyceride levels in blood than non-carriers (AA genotype) in concordant with the result of blood pressure. Elevated plasma triglyceride levels are observed in many metabolic diseases such as metabolic syndrome, diabetes mellitus and hypertension (Austin, 1999, Brewer, 1999), and in diabetic model mice PTGS2 gene expression is previously reported to be increased (Laybutt et al., 2002).

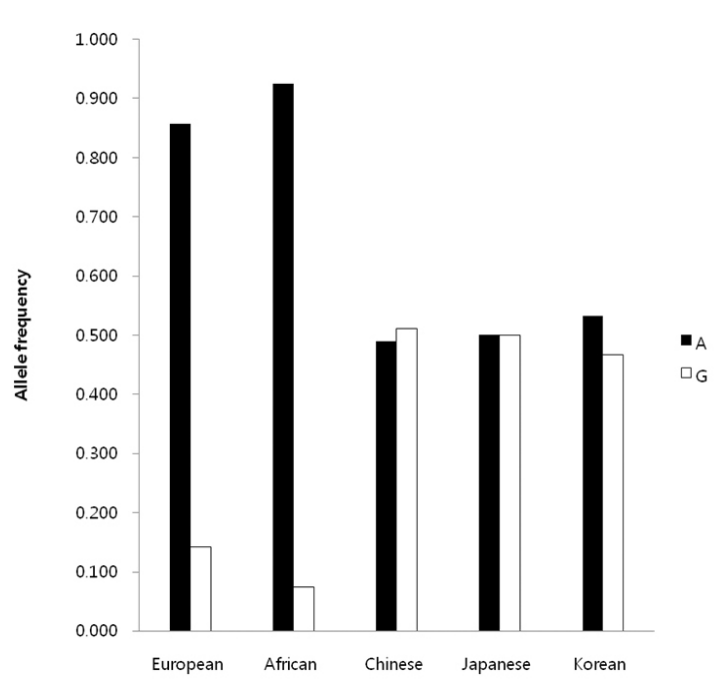

Fig. 2. Genotype frequencies of PTGS2 (-1329A $>\mathrm{G}$ : rs689466) among different population groups. Genotype frequencies except Korean were obtained from HapMap data (http://www.ncbi.nlm.nih.gov/SNP/).

Table 3. Linear regression analysis between PTGS2 genotypes and blood pressure adjusted by age, sex and BMI

\begin{tabular}{|c|c|c|c|c|c|c|}
\hline & \multicolumn{3}{|c|}{ Individual number of genotype (mean $\pm \mathrm{SD} \mathrm{mmHg}$ ) } & \multirow{2}{*}{$\begin{array}{c}\text { Co-dominant } \\
\mathrm{p}\end{array}$} & \multirow{2}{*}{$\frac{\text { Dominant }}{p}$} & \multirow{2}{*}{$\frac{\text { Recessive }}{p}$} \\
\hline & $\mathrm{C} / \mathrm{C}$ & $\mathrm{C} / \mathrm{R}$ & $R / R$ & & & \\
\hline \multicolumn{7}{|c|}{ rs689466 (-1329A > G, Promoter) } \\
\hline SBP & $132(118.18 \pm 15.87)$ & $233(122.25 \pm 17.93)$ & $102(121.67 \pm 17.39)$ & 0.038 & 0.008 & 0.51 \\
\hline DBP & $132(73.51 \pm 9.08)$ & $233(76.01 \pm 10.13)$ & $102(76.16 \pm 10.09)$ & 0.018 & 0.006 & 0.302 \\
\hline \multicolumn{7}{|c|}{ rs5275 (+6365T > C, Exon10, 3'UTR) } \\
\hline SBP & $283(120.75 \pm 17.98)$ & $156(121.61 \pm 16.21)$ & $28(119.6 \pm 16.69)$ & 0.792 & 0.926 & 0.631 \\
\hline DBP & $283(75.46 \pm 10.63)$ & $156(75.03 \pm 8.71)$ & $28(75.88 \pm 8.39)$ & 0.667 & 0.497 & 0.767 \\
\hline \multicolumn{7}{|c|}{ rs4648308 (+8806G >A, 3'Downstream) } \\
\hline SBP & $418(120.92 \pm 17.09)$ & $50(122.07 \pm 19.11)$ & $1(108.67 \pm)$ & 0.935 & 0.994 & 0.535 \\
\hline DBP & $418(75.41 \pm 10.09)$ & $50(75.04 \pm 7.98)$ & $1(69.33 \pm)$ & 0.555 & 0.595 & 0.605 \\
\hline
\end{tabular}

Bold indicated significant result by $p$-value $<0.05$.

SBP: systolic blood pressure, DBP: diastolic blood pressure. 
Human DNA sequence of $1 \mathrm{~kb}$ promoter region containing rs689466 was compared with those of other vertebrates, which revealed $98 \%$ homology in chimpanzee and $92 \%$ in rhesus macaque, but no homologies were found in mouse and rat. Furthermore the transcription factor analysis using TRANSFAC database (http:www. cbrc.jp/research/db/TFSEARCH.html) found that $v-M y b$ transcription factor binding site was disappeared by the substitution of A allele with $G$ allele in rs689466. According to these results, SNP rs689466 might be important site for the gene expression regulation of PTGS2 on primates.

Genetic association studies often fail to replicate previous findings, in part due to the polygenetic nature of the disease. Another potential reason may be the diversity of investigated populations. Allele frequencies of PTGS2 (rs689466:-1329A > G) in Korean population were compared with those in other populations obtained from HapMap data (Fig. 2). Asian populations have higher allele frequencies (Korean; 0.467, Japanese; 0.500, Chinese; 0.511) than European and African populations (European; 0.142, African; 0.075). SNP rs689466 was found to be insignificant in the hypertension results of Genome-wide association study of Welcome Trust Case Control Consortium (http://www.wtccc.org.uk/) that might be attributed by the low allele frequency of the SNP.

In this study, we investigated the effect of PTGS2 polymorphisms on the blood pressure and lipids in healthy subjects selected from Ansung and Ansan community cohort and identified one polymorphism of PTGS2 gene which is associated with blood pressure as well as triglyceride in blood. This finding sheds light on a genetic polymorphism of PTGS2, rs689466 as a useful genetic marker for the blood pressure and blood triglyceride level.

\section{Acknowledgements}

This study was supported by an intramural grant from the National Institute of Health, Korea and an intramural grant from Kyung Hee University.

\section{References}

Abassi, Z., Brodsky, S., Gealekman, O., Rubinstein, I., Hoffman, A., and Winaver, J. (2001). Intrarenal expression and distribution of cyclooxygenase isoforms in rats with experimental heart failure. Am. J. Physiol. Renal Physiol. 280, F43-53.

Adderley, S.R., and Fitzgerald, D.J. (1999). Oxidative damage of cardiomyocytes is limited by extracellular regulated kinases 1/2-mediated induction of cyclooxygenase-2. J. Biol. Chem. 274, 5038-5046.

Anderson, R.J., Berl, T., McDonald, K.M., and Schrier, R.W.
(1976). Prostaglandins: effects on blood pressure, renal blood flow, sodium and water excretion. Kidney Int. 10, 205-215.

Austin, M.A. (1999). Epidemiology of hypertriglyceridemia and cardiovascular disease. Am. J. Cardiol. 83, 13F-16F.

Barrett, J.C., Fry, B., Maller, J., and Daly, M.J. (2005). Haploview: analysis and visualization of LD and haplotype maps. Bioinformatics. 21, 263-265.

Brewer, H.B., Jr. (1999). Hypertriglyceridemia: changes in the plasma lipoproteins associated with an increased risk of cardiovascular disease. Am. J. Cardiol. 83, 3F-12F.

Daniels, E.G., Hinman, J.W., Leach, B.E., and Muirhead, E.E. (1967). Identification of prostaglandin E2 as the principal vasodepressor lipid of rabbit renal medulla. Nature 215, 1298-1299.

Dennis, L.K., Eugene, B., Stephen, H., Dan, L., Larry, J.J., and Anthony, S.F. (2004). Harrison's principles of internal medicine. 16th edition. (McGraw-Hill Professional)

Laybutt, D.R., Sharma, A., Sgroi, D.C., Gaudet, J., Bonner-Weir, S., and Weir, G.C. (2002). Genetic regulation of metabolic pathways in $\beta$-cells disrupted by hyperglycemia. J. Biol. Chem. 277, 10912-10921.

Hao, C.M., and Breyer, M.D., (2008). Physiological regulation of prostaglandins in the kidney. Annu. Rev. Physiol. 70, 357-377.

Hla, T., and Neilson, K. (1992). Human cyclooxygenase-2 cDNA. Proc. Natl. Acad. Sci. U. S. A. 89, 7384-7388.

Iwai, N., Tago, N., Yasui, N., Kokubo, Y., Inamoto, N., Tomoike, H., and Shioji, K. (2004). Genetic analysis of 22 candidate genes for hypertension in the Japanese population. J. Hypertens. 22, 1119-1126.

Livak, K.J. (1999). Allelic discrimination using fluorogenic probes and the $5^{\prime}$ nuclease assay. Genet Anal. 14, 143-149.

Naber, C.K., and Siffert, W. (2004). Genetics of human arterial hypertension. Minerva Med. 95, 347-356.

Nakayama, T., Soma, M., Watanabe, Y., Hasimu, B., Sato, M., Aoi, N., Kosuge, K., Kanmatsuse, K., Kokubun, S., Marrow, J.D., and Oates, J.A. (2002). Splicing mutation of the prostacyclin synthase gene in a family associated with hypertension. Biochem. Biophys. Res. Commun. 297, 1135-1139.

Nickerson, D.A., Tobe, V.O., and Taylor, S.L. (1997). PolyPhred: automating the detection and genotyping of single nucleotide substitutions using fluorescence-based resequencing. Nucleic Acids Res. 25, 2745-2751.

Oh, S.H., NamKung, J.H., and Park, T.S. (2007). Regression models for haplotype-based association studies. Genomics \& Informatics 5, 19-23.

Park, H.S., Kim, J.I., Cho, S.I., Sung, J.H., Kim, H.L., Ju, Y.S., Bayasgalan, G., Lee, M.K., and Seo, J.S. (2008). Genome-wide linkage study for plasma hdl cholesterol level in an isolated population of mongolia. Genomics \& Informatics 6, 8-13.

Rozen, S., and Skaletsky, H. (2000). Primer3 on the WWW for general users and for biologist programmers. Methods Mol. Biol. 132, 365-386.

Tazawa, R., Xu, X.M., Wu, K.K., and Wang, L.H. (1994). Characterization of the genomic structure, chromosomal 
location and promoter of human prostaglandin $\mathrm{H}$ synthase-2 gene. Biochem. Biophys. Res Commun. 203, 190-199

Wong, S.C., Fukuchi, M., Melnyk, P., Rodger, I., and Giaid, A. (1998). Induction of cyclooxygenase-2 and activation of nuclear factor-kappaB in myocardium of patients with congestive heart failure. Circulation 98, 100-103.

Xu, X., Rogus, J.J., Terwedow, H.A., Yang, J., Wang, Z., Chen, C., Niu, T., Wang, B., Xu, H., Weiss, S., Schork, N.J., and Fang, Z. (1999). An extreme-sib-pair genome scan for genes regulating blood pressure. Am. J. Hum. Genet. 64, 1694-1701.

Zewde, T., and Mattson, D.L. (2004). Inhibition of cyclooxygenase-2 in the rat renal medulla leads to sodium-sensitive hypertension. Hypertension 44, 424-428.

Zhang, J., Ding, E.L., and Song, Y. (2006). Adverse effects of cyclooxygenase 2 inhibitors on renal and arrhythmia events: meta-analysis of randomized trials. JAMA. 296, 1619-1632. 
116 Genomics \& Informatics Vol. 6(3) 110-116, September 2008

Supplemental Table 1. Sequences of the amplifying primers used for direct sequencing of PTGS2 gene

\begin{tabular}{clll}
\hline Forward Primer & \multicolumn{1}{c}{ Sequence } & Reverse Primer & \multicolumn{1}{c}{ Sequence } \\
\hline PTGS2_1F & CCGTGTCTCATGAAGAATCA & PTGS2_1R & GGCGATGGCCAGAATTT \\
PTGS2_2F & GGACATTTAGCGTCCCTGC & PTGS2_2R & GGTTTCCGCCAGATGTCTT \\
PTGS2_3F & GCAAAGACTGCGAAGAAGAA & PTGS2_3R & AGCTCTTTCCCAAGTCACG \\
PTGS2_4F & TCCATTCTAAGGCAGGTTAAAAA & PTGS2_4R & TTGGCGATTAAGATGGAAGG \\
PTGS2_5F & CCTGAAAAATCAATATTGCCA & PTGS2_5R & CAAGAAAGGAGATGGTGACTG \\
PTGS2_6F & GCAAATGAGCGTCTTGGTAT & PTGS2_6R & GCGGCATAATCATGGTACA \\
PTGS2_7F & TCAGTTTGTAGCTTTGGTGGA & PTGS2_7R & GCAACTGGAATGCAATTTTA \\
PTGS2_8F & TGACAAGGAAGAAAACAGAAATGA & PTGS2_8R & AAATTCAATGGGACACCAGC \\
PTGS2_9F & CTGGTGTCCCATTGAATTT & PTGS2_9R & CCATCTCGAAAAGAAACCA \\
PTGS2_10F & CTGGCCCCTAAACTTCTTAAA & PTGS2_10R & CGCAACAGGAGTACTGACTTC \\
PTGS2_11F & ATCAATGCAAGTTCTTCCCG & PTGS2_11R & TCCAAGACAGCTCTTTTGGT \\
PTGS2_12F & TCACCTGTAAAAGCTTGTTTGATT & PTGS2_12R & AGGAACAGCATGCAGGTAGC \\
PTGS2_13F & TTGCAAAAGTAGCAATGACCTC & PTGS2_13R & TCAGTGACAATGAGATGTGGAAA \\
PTGS2_14F & TTCTTTTCCACATCTCATTGTCA & PTGS2_14R & ACATTCGCATACACAACCCA \\
PTGS2_15F & TTCAGTGCCTCAGACAAATG & PTGS2_15R & AAGATTTTGAAAGTGGTGCTG \\
\hline
\end{tabular}

Supplemental Table 2. Polymorphisms discovered in PTGS2 genes

\begin{tabular}{llll}
\hline dbSNP & \multicolumn{1}{c}{ Position } & Polymorphism & Minor allele Frequency \\
\hline rs689465 & promoter & $-1424 \mathrm{~A}>\mathrm{G}$ & 0.042 \\
rs689466 & promoter & $-1329 \mathrm{~A}>\mathrm{G}$ & 0.467 \\
rs20417 & promoter & $-899 \mathrm{G}>\mathrm{C}$ & 0.043 \\
novel & promoter & -788 TAGinsdel & 0.022 \\
rs5270 & promoter & $-297 \mathrm{c}>\mathrm{G}$ & 0.021 \\
rs4648257 & Exon 1 (5'utr) & $-118 \mathrm{~T}>\mathrm{A}$ & 0.021 \\
rs4648258 & intron 1 & $+115 \mathrm{C}>\mathrm{G}$ & 0.021 \\
rs2066824 & intron 2 & $+1051 \mathrm{C}>\mathrm{A}$ & 0.021 \\
rs5277 & Exon 3 & $+1226 \mathrm{G}>\mathrm{C}$ V102V & 0.125 \\
rs2066823 & intron 3 & $+1266 \mathrm{C}>\mathrm{T}$ & 0.021 \\
rs4648263 & intron 3 & $+1350 \mathrm{G}>\mathrm{C}$ & 0.021 \\
rs20428 & intron 3 & $+1814 \mathrm{~T}>\mathrm{C}$ & 0.021 \\
rs3218622 & Exon 6 & $+3418 \mathrm{G}>\mathrm{A} \mathrm{R228H}$ & 0.021 \\
rs2066826 & intron 6 & $+3496 \mathrm{G}>\mathrm{A}$ & 0.021 \\
rs2066825 & intron 6 & $+3568 \mathrm{Ainsdel}$ & 0.021 \\
rs3218623 & Exon 8 & $+4141 \mathrm{G}>\mathrm{A} \mathrm{V355V}$ & 0.023 \\
rs3218624 & Exon 8 & $+4336 \mathrm{G}>\mathrm{A}$ L400L & 0.023 \\
rs3218625 & Exon 10 & $+5882 \mathrm{G}>\mathrm{A} \mathrm{G587R}$ & 0.021 \\
rs5275 & Exon 10 (3'utr) & $+6365 \mathrm{~T}>\mathrm{C}$ & 0.226 \\
rs4648296 & Exon 10 (3'utr) & $+7355 \mathrm{~T}>\mathrm{C}$ & 0.023 \\
rs4648297 & Exon 10 (3'utr) & $+7364 \mathrm{G}>\mathrm{A}$ & 0.023 \\
rs4648303 & Exon 10 (3'utr) & $+8225 \mathrm{C}>\mathrm{T}$ & 0.028 \\
rs4648306 & Exon 10 (3'flanking) & $+8719 \mathrm{G}>\mathrm{A}$ & 0.021 \\
rs4648307 & Exon 10 (3'flanking) & $+8720 \mathrm{C}>\mathrm{A}$ & 0.021 \\
rs4648308 & Exon 10 (3'flanking) & $+8806 \mathrm{G}>\mathrm{A}$ & 0.055 \\
\hline & & & \\
\end{tabular}

